Chirurg 2016 $87: 252$

DOI 10.1007/s00104-016-0167-4

Online publiziert: 24. Februar 2016

(c) Springer-Verlag Berlin Heidelberg 2016

CrossMark

O. Strobel · M. W. Büchler

Klinik für Allgemein-, Viszeral- und Transplantationschirurgie, Universität Heidelberg, Heidelberg, Deutschland

\title{
Pankreaschirurgie in Deutschland: Bessere Ergebnisse bei hohen Fallzahlen
}

Korrektur für bekannte Einflussfaktoren untersucht. Als primärer Zielparameter wurde die 1-Jahres-Mortalität, als sekundäre Zielparameter wurden die Krankenhaus- und 90-Tage-Mortalität untersucht.

Hintergrund und Fragestellung. Bei komplexen chirurgischen Eingriffen ist eine hohe Erfahrung notwendig, um gute Ergebnisse zu erzielen. Für die Pankreaschirurgie konnte in mehreren Studien aus dem Ausland gezeigt werden, dass sowohl die perioperative als auch die Langzeitmortalität mit zunehmender Fallzahl sinkt. Dies hat zur Diskussion über notwendige Mindestmengen zur Qualitätssicherung in der Pankreaschirurgie geführt. Eine entsprechende Datenengrundlage aus Deutschland fehlt jedoch bisher. Alsfasser et al. untersuchten nun deutschlandweit die Beziehung zwischen Fallzahl und Mortalität in der Pankreaschirurgie.

Methoden. In der Studie wurden die administrativen Falldaten der Allgemeinen Ortskrankenkassen (AOK), die etwa $30 \%$ der stationären Fälle abdecken, deutschlandweit anonymisiert ausgewertet. Eingeschlossen wurden alle elektiven und notfallmäßigen Pankreasoperationen von 2008 bis 2010, wenn nicht innerhalb des letzten Jahres bereits einmal eine Pankreasoperation durchgeführt worden war. Die Gesamt-Fallzahlen der Krankenhäuser wurden anhand der Fallzahlen von AOK-Patienten in den Jahren 2008 bis 2010 extrapoliert. Die Beziehung zwischen Fallzahl und Behandlungsergebnis wurde mittels multipler logistischer Regressionsanalysen unter
Ergebnisse. Von 9566 Patienten wurden 46,1\% wegen eines Pankreaskarzinoms, $16,2 \%$ wegen einer chronischen Pankreatitis, 8,7\% wegen einer akuten Pankreatitis und 28,9\% wegen anderer Erkrankungen operiert. Krankenhaus-, 90-Tage- und 1-Jahres-Mortalität waren $10,1,12,1$ und 29,8\%. Die nicht risikoadjustierte 1-Jahres-Mortalität nahm von $34,4 \%$ bei den Krankenhäusern in der Quintile mit der geringsten Fallzahl (1-12 extrapolierte Fälle) schrittweise ab bis $\mathrm{zu} 23,3 \%$ in der Quintile mit den höchsten Fallzahlen (85-294 Fälle). Auch die risikoadjustierte 1-Jahres-Mortalität war in den 3 Quintilen mit niedrigerer Fallzahl mit Odds-Ratios von 1,73, 1,53 und 1,37 signifikant erhöht im Vergleich zu den Krankenhäusern in der Quintile mit den höchsten Fallzahlen. Bei der Krankenhaus- und 90-Tage-Mortalität zeigten sich vergleichbare Ergebnisse. Der Effekt der Fallzahl auf die Mortalität war vergleichbar mit dem Einfluss relevanter kardialer, pulmonaler und renaler Begleiterkrankungen.

Diskussion. Die Autoren folgern, dass diese Ergebnisse dafür sprechen, Pankreaschirurgie nur an Krankenhäusern mit genügend hoher Fallzahl, Expertise und Ressourcen durchzuführen.

Während der Zusammenhang zwischen Fallzahl und Ergebnisqualität insbesondere für die Pankreaschirurgie bereits in früheren Studien klar dargelegt wurde, ist die aktuelle Studie in mehrfacher Hinsicht relevant:

- Die Studie zeigt, dass Pankreaschirurgie in der Breite und in Deutschland nach wie vor mit einer hohen Krankenhausmortalität (> $10 \%$ ) assoziiert bleibt, die nicht angesichts der publizierten Daten einiger spezialisierter Zentren unterschätzt werden darf.

- Volumen-Outcome-Beziehungen in der Chirurgie können populations-, kultur- und systembedingt variieren. Die vorliegende Studie belegt anhand einer großen repräsentativen Stichprobe klar, dass das Mortalitätsrisiko in der Pankreaschirurgie in Deutschland bei niedriger Fallzahl signifikant und relevant erhöht ist.

Diese Fakten sollten bei der Indikationsstellung für eine Pankreasoperation berücksichtigt werden, insbesondere bei Patienten, die aufgrund Ihrer Begleiterkrankungen bereits ein erhöhtes Mortalitätsrisiko haben.

\section{Korrespondenzadresse}

\section{PD Dr. O. Strobel}

Klinik für Allgemein-, Viszeral- und Transplantationschirurgie, Universität Heidelberg

Im Neuenheimer Feld 110, 69120 Heidelberg, Deutschland

Oliver.Strobel@med.uni-heidelberg.de

Interessenkonflikt. O.Strobel und M.W. Büchler geben an, dass kein Interessenkonflikt besteht. 